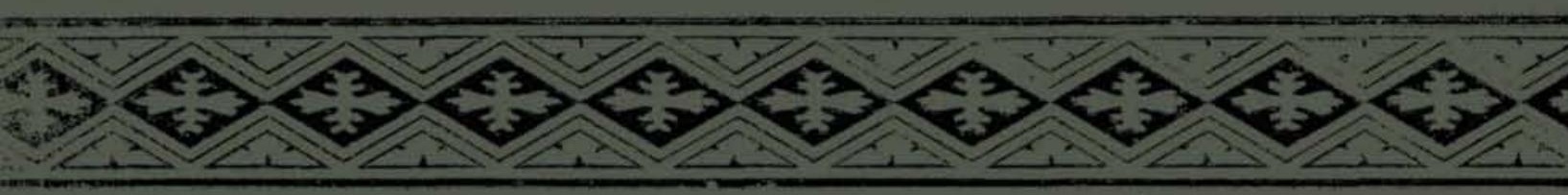

\author{
THE \\ C L A S S I A L \\ R E V I E W
}

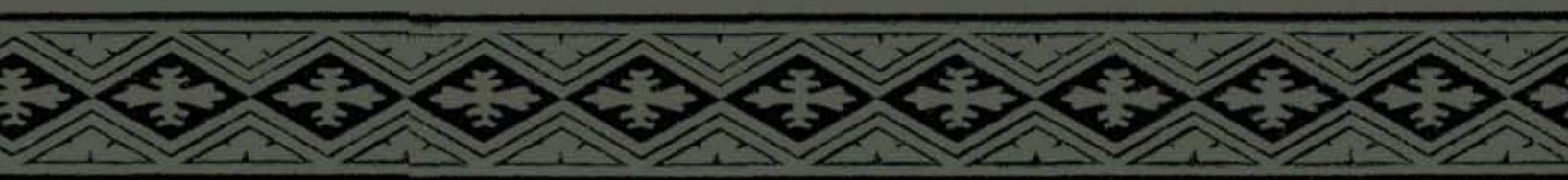


THE

C L A S S I C A L

\section{REVIEW.}

\section{VOLUME V.}

Pondon :

DAVID NUTT, 270 AND 271 STRAND, 1891.

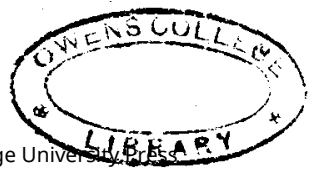


Richard Clay and Sons, Limited, LONDON AND BUNGAY. 


\title{
TABLE OF CON'TEN'TS.
}

\author{
Nos. 1 and 2.
}

J. G. Frazer. Swallows in the house $\stackrel{\text { Page }}{1}$

F. Carter and M. W. Humphreys. On some uses of the Aorist Participle.

A. Palmer. Catulliana . . . . . . .

W. M. Lindsay. Notes on Festus and Nonius . . . . . . . . . .

T. K. Aввотr. On the quotations from the Old Testament in the Fourth Gospel . . . . . . . . . . 11

P. Schwenke. Apparatus Criticus to Cicero De Natura Deorum . . . . . 12

F. F. Аввотт. Notes upon Latin Hybrids 18

Westcott's Epistle to the Hebrews. J. H. Thayer . . . . . . . . . 18

Hude's Thucydides VI.-VIII. E. C. MarchaNT ........ 22

Westermann's Demosthenes, Vol. III. W. WAYTE ........ 24

Headlam's Meleager. H. Babington Sмiтн . . . . . . . . . . . . . 26

Melber's Dio Cassius, Vol. I. G. Mc. N. RushFoRTH . . . . . . . . . 27

Kaibel's Athenaeus. J. B. BuRY . . . 29

Schmid on the Later Atticism. J. E. SandYs . . . . . . . . . . . 31

Fierville's Quintilian. W. Peterson : 32

Bauer's Silius Italicus. W. E. Heitrand 36

De Vries' Studies in Palaeography. Robinson Ellis . . . . . . . 38

Peck's Suetonius. A. T. Murray . . 38 Otto's Roman Proverbs. W. RHYs RoBerTs .......... . 41

Schwabe's edition of Teuffel's Roman Literature. G. C. W ARR . . . . 42

Two Books on the Rigveda. E. V. AnNold 43

Frazer's Golden Bough. W. W. FowLER 48

Schmidt's Corcyrean Studies. H. F.

Tozer . . . . . . . . . . . 53

Rhode on the Tunny Fish. H. F. Tozer 54

Mahaffy's Greek Pictures. H. F. Tozer 55

Mahaff'y's Greek World under Roman Sway. C. Torr . . . . . . . . . 56

New Editions of Kraner's Caesar. F. Haverfield . . . . . . . . 58

Oehler's Illustrations to Caesar Bell. Gall. F. H. . . . . . . . . 58
W. F. Allen's Annals of Tacitus. S. ${ }^{\text {PAGI }}$ Hart . . . . . . . . . . 58 W. F. Allen's Roman History. S. HarT 59 Hime's Introduction to the Latin Language. J. E. NixoN . . . . . 59 Peck's Latin Pronunciation. F. D. Allen . . . . . . . . . . . 60 R. Fisch on Latin Personals in -O. H. N. . . . . . . . . . 61 Commentationes Fleckeisenianae. J. B. Bury . . . . . . . . . . . . . 62 Meister's Greek Dialects. H. W. SMYTH 62 Seger's Nikephoros Bryennios. J. B. BuRY . . . . . . . . . . . 63

C. E. Bishop on verbals in -tos in Aeschylus. A. Fairbanks . . . . . . 63 Correspondence.

A. Sidgwick on Mr. Page's Review of Papillon and Haigh's Aeneid . . 64 Verse Translations by E. D. S., F. HAvERFIELD and C. E. S. HEAdLaM . . $65 \& 66$ Notes.

$\kappa a \nu \theta \eta \dot{\lambda} \lambda$. W. LORING . . . . . . 66 Soph. Aj. 651. R. Whitelaw . . . 66 Thuc. II. 11. F. HaverFIELD . . . 67 Tragic Tribrachs. F. Haverfield . 67 The Latin Aorist Subjunctive. F. A. Kinkpatrick, W. H. Bocock . . 67 Spartian. Viț. Hadr. XI. E. N. BenNetT . . . . . . . . . 68

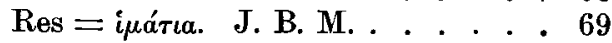
The Upright Stone at Kilmalkedar . 69 The Newly-discovered Treatise of Aristotle . . . . . . . . . . . . 69 Monthly Record . . . . . . . . 72 Archaeology.

Ephemeris Epigraphica. H. F. PeLHAM . . . . . . . . . . . . . 74 Müllenhoff's Deutsche Altertumskunde. C. W. Boase . . . . 75 An early Graeco-Egyptian bilingual dedication. C. SмIтH . . . . . . 77

Aus der Anomia. C. Sмiтh . . . . 79 Sunmaries .. . . . . . . . 80 Bibliography .. . . . . . . 85 
No. 3.

The New Aristotle Papyrus in its bearing on Textual Criticism. W. G. Rutherford . . . . . . . . . . . 89 Ovidiana. A. Palmer . . . . . . . . 92 Etymology of Osteria. F. F. Аввотт . 95 Dr. Dörpfeld on the Greek Stage. H. Richakds . . . . . . . . 97

Verrall's Ion. A. Goodwin . . . . . 97 Shuckburgh's Herodotus V. Ev. Aввотт 99 Tyrrell and Purser's Letters of Cicero.

G. E. JEans . . . . . . . . 100

Palaiphatos, a Rationalist of the Fourth Century B.c. J. B. BuRY . . . . 102 Schubert's Ajax. E. B. England . . 103 Usener's Theodosius. A. Plummer . . 103 Dübner's Sulpicius Severus. J. $H$. Lup'Ton . . . . . . . . . . . . 103 Collectio Juris Antejustiniani. E. C. Clark .......... . . 104 Bousfield's Latin Verse. H. Kynaston 104 Aristotle on the Constitution of Athens :-

Emendations, pp. 105-119 :

Notes by C. Tork, L. Campbell, J. E. Sandys, J. E. B. Mayon, H. JACKson, H. Richards, W. WYSE . 119-122
Un-Aristotelian Words and Phrases in, J. B. MAYOR . . . . . . . . . 122

The New Antiope Fragments . . . 123

Emendations by W. G. Rutherford and L. CampBeLL , . . . . . . . 124 Notes.

Leucon's Liberality to Athens. W. Hobrouse and C. Torr . . . . 127

Eur. Bacch. 506. J. B. Bury . . . 127 Eur. El. 797. C. H. KEENE . . . . 127 Thuc. II. 4, 4. E. C. Marchant . . 128 Eic. ad Att. I. 16 . . . . . . . 128 Verg. Aen. X. 1. W. J. Evans . . 128 Caper p. 103. W. M. LindsaY . . . 128

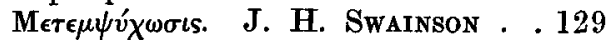

Stanko. J. H. Abrahall . . . . 129 Monthly Record.

Archaeology.

The House at Delos. F. B. TARBell 130 Reinach's Bibliothèque des Monuments

Figurés, Vol. II. . . . . . . . . 131

A Stele in the British Museum. A. S. M. . . . . . . . . . 132

Excavations at Chester . . . . . 132 Summaries. . . . . . . . . . . 133 Bibliography. . . . . . . . . . 135

\section{No. 4.}

G. S. SALE : Notes on Horace . . . 137

A. Palmer : Horatiana . . . . . 139

J. A. Cross: Quotations in the Fourth Gospel . . . . . . . . . . 142

P. Schwenke : Apparatus Criticus to Cicero. N.D. . . . . . . . . . . 143

Jebb's Philoctetes. A. S. . . . . . . 147

Gwatkin and Shuckburgh's Edition of 'Aeschines in Ctesiphonta.' J. H. WRIGHT .......... 149

Bywater's Ethics. H. Richards . . . 152 Aristotle's Constitution of Athens. W.

L. Newman ... . . . . . 155

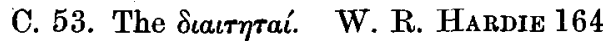

C. 44. The Deposition of Pericles. E. C. Marchan'T . . . . . . . 165

C. 4. Constitution of Draco. J. W. Headlam . . . . . . . 166

C. $22,23,28$. L. WHIBLEY . . . . 168

C. 25. Themistocles. G. F. HidL . . 169

C. 52. The Eleven . . . . . . 169
Wickham's Horace, Vol. II. R. Y. TYRReLL . . . . . . . . . . . . . 169 Eton Latin Grammar, Ed. 2. E. A. SonNenschein . . . . . . . . . . 172 Notes on the Constitution of Athens.

Emendations, pp. 175-182 :

Alphabet, p. 183 :

Un-Aristotelian Words and Phrases. H. Richards, J. B. Mayor, A. PlatT . . . . . . . . 184 New Fragments of the Antiope. W. Headiam, J. E. Sandys, R. Garnett . 185 Notes.

Aen. VI. 743. R. Whitelaw . . 186 Soph. Aj. 651. G. E. Marindin . . 186 Correspondence.

J. P. Mahafy . . . . . . . 186

Monthly Record . . . . . . . . . 187

Summaries . . . . . . . . . . 189

Bibliography . . . . . . . . 191 
No. 5.

W. R. Hardie: Allusive Language in

F. CARTER: Notes on Thucydides IV. . 195

E. H. Mrles : Latin Infinitive Passive. 198

P. Schwenke : Apparatus Criticus on Cicero. N. D. . . . . . . . . 200 Sonnenschein's Rudens. RoBinson ElLIs 205 Goetz' Miles Gloriosus. R. Y. TyrRell 206 Benoist's Virgil. T. E. Page . . . . 208 Jacobs' Fables of Aesop. F. B. Jevons 212 Hilgard's Greek Grammarians. E. G. SIHLER. . . . . . . . . 215

Blass, Pronunciation of Ancient Greek, English Translation. B. I. WheELer 217

Wharton's Etyma Latina. H. D. DARBISHIRE . . . . . . . . . . . . 217

Merriam's Telegraphing among the Ancients. J. G. . . . . . . . 220

Three Elementary Books on Greek . . 220

The Constitution of Athens:-

Attic Phratries. W. R. Paton . . 221

The Authorship. L. WHIBLeY . . . 223

Miscellaneous. E. S. 'Тномpson, W. Wyse, H. Richards . . . . . . 224

Un-Aristotelian Words. E. J. CHINNOCK . . . . . . . . . . 229 Greek Lyric Poetry . . . . . . 193

Notes.

Soph. Aj. 651, again. R. Whitelaw 230

Swallows in the House. J. G. Frazer, D'Arcy W. Thompson, C. Harper 230 Arist. Post. Anal. I. 5, 2. C. S.

Adamson and H. Richards . . . 231

Verg. Georg. I. 316. H. Richards . 232

Verg. Aen. VI. 567. G. Mc N. R. . 232

Antiope. M. R. JAMES . . . . . 232

Hiberno-Saxon Symbol for Autem.

J. E. SANDYS . . . . . . . 232

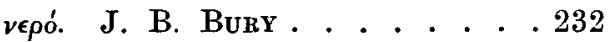

Correspondence.

Cecil Torr . . . . . . . . . . . 232

Monthly Record . . . . . . . . . 233

Tozer's Islands of the Aegean. W. R. Paton ........ 237

Theatre of Megalopolis. L. Dyen, E.

Sellers . . . . . . . . . . . . . 238

Re-excavated Relics. F. Haterifield . 240

Acquisitions of the British Museum.

A. S. M. . . . . . . . . . . . . 241

A Vase-painting. W. WATKISs LloYd 241

A Carthaginian Ambassador in a Greek

Inscription. J. Hoskyns Abrahall . 242

Summaries of Periodicals . . . . . . 242

Bibliography . . . . . . . . 243

No. 6.

J. A. Cross: Hours of the Day in the Fourth Gospel . . . . . . . . 245

R. Whitelaw and F. Carter : On Some Uses of the Aorist Participle . . 248

F. G. Plaistowe : On Sonant Z . . . 253

Campbell's Aeschylus. A. W. Polland 255

Dakyns' Translation of Xenophon. E. S. Shuckburah . . . . . . . . 257

Aristophanes' Use of Prepositions. E. C. Marchant . . . . . . . . . . 258

Some French Books on Palaeography. E. Maunde Thompson ..... 261

Stowasser's Dunkle Wörter. H. NETTLESHIP . . . . . . . . . . . . . . 263

Studies by Studemund's Pupils. E. A. SoNNENSCHEIN . . . . . . . . . 264

Montague's Selected Letters of Cicero. F. F. Аввотт . . . . . . . . . . 266

Stephenson's Livy, Book XXVII. M. T. Tatham . . . . . . . . 266

Dr. Weymouth on the Greek Aorist and Perfect . . . . . . . . . 267

Correspondence.

Dr. Verrall on the Fire-Signals in the Agamemnon . . . . . . . . . 269

The Constitution of Athens.

New Readings of the MSS. F. G. KENYON
On the Use of the Hiatus. J. W.

Hraduam . . . . . . . . . . 270

Un-Aristotelian Words and Phrases.

H. Richards . . . . . . . . . 272

Emendations by J. R. WARdale . . 273

Emendations by Foreign Scholars . . 274

Further Notes by W. Wyse, E. S.

Thompson, C. Tork, C. Smith . . 274 Notes.

A Carthaginian Ambassador. $\quad$ C. T. . 278

Plato Rep. 532 B. $\quad$ E. J. Palmer . . 278

Hor. $E$ p. I. ii. 31 . A. S. O. . . . . 278

Ov. R.A. 207. E. D. S. . . . . 278

Ov. Trist. III. xi. 33. P. SAndford 279

The Exile of Juvenal. H. A. STrong 279

Wilkins' Roman Literature. W. K.

Clement . . . . . . . . . . . 279

Danger of Quoting from Memory. G.

E. Jeans . . . . . . . . 279

Archaeology.

The Harbours of Carthage. C. Toke 280

Theatre of Megalopolis . . . . 284

Summaries . . . . . . . . . 285

Monthly Record . . . . . . . . 287

Summaries of Periodicals . . . . . . 289

Bibliography. . . . . . . . 290
Arist. Ran. 1433. H. Richards . . 278 


\section{No 7.}

A. E. Housman : Adversaria Orthographica . . . . . . . . . 293

R. Seryour Conway : Origin of the Latin Gerund and Gerundive . . . . 296

J. P. Postgate ; Latin Future Infinitive in -turum . . . . . . . . . 301

F. T. Tarbell : Greek Deliberative Subjunctive in Relative Clauses . . . . . 302

P. Schwenke : Apparatus Criticus on Cicero. N. D. . . . . . . . . . . 302

Bury's Nemean Odes. J. E. SANDYs . . 305 Merry's Edition of the Birds. R. Y. Tyrrell . . . . . . . . . . . . 309

Demosthenes De Corona, by F. Blass. S. H. Butcher . . . . . . . . . 309

Plutarch's Lives of Galba and Otho, by E. G. Hardy. H. Holden . . . 315

Epigrams of Crinagoras. J. H. WrIGHT 317

Krumbacher's History of Byzantine Literature. J. B. BURY . . . . . . 318

Rossbach and Westphal's Greek Metres.

C. B. Herberden . . . . . . . . 320

Schoell's Casina. E. A. Sonnenschein . 321

Peskett's Caesar B.C., Book I. B. Perrin . . . . . . . . . . . . . 324

Whibley's Livy, Book V. H. M. Stephenson . . . . . . . . . . 325

Lindsay's Juvenal. M. H. MorgaN . 326 Scott's Translation of Calpurnius. E. D. A. Morshead . . . . . . . . . 327 Autenrieth's Homeric Dictionary. T. D. Seymour . . . . . . . . . . 328

Gelzer's Georgius Cyprius. F. HaverFIELD . . . . . . . . . . . . 328
Etienne, La Langue Francaise ${ }^{\text {PAgE }}$ SPEnCER .......... . 329 Goyau's Chronologie de l'Empire Romain.

E. G. HARDY . . . . . . . . . 329

Perry's Greek Literature. B. Perrin . 330 Fagan's British Museum . . . . . 331 Greek Elegiacs. L. CampbelL . . . . 331

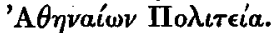

Recent Literature. F. G. K. . . . 332

A Reply to Professor Gomperz. $\mathbf{H}$.

RichaRds . . . . . . . . . . 333

Further Notes by H. Richards, W.

Wyse, E. S. Thompson, E. Poste . . 334 Notes.

Pindar Nem. IX. 22. A. C. Pearson 337 Virg. Aen. VI. 567, III. 702. A.

Platt . . . . . . . . . 337

Hor. C. II. xi. 21. P. SANDFord . . 337

Hor. Ep. I. vii. 29. H. A. Strong . 337

Ne ó. W. Leaf . . . . . . . 338

Reply to Professor Clement. A. S.

WILKINS . . . . . . . . . . 338

Telegraphing among the Ancients.

A. C. Merriam, J. G., \& W. R. Paton 338

Split Totems. A. Platt . . . . . 339

Archaeology.

Monthly Record. H. B. W ALters . 339

Acquisitions of the British Museum (1-5), A. S. Murray, (6) C. SMith . 341

The Greek Theatre. A. C. Merriam,

Miss Sellers, Editor. . . . . . 343

A Megaric Inscription. M. L. Earle 344 Summaries of Periodieals . . . . . 345 Bibliography . . . . . . . . . 347

No. 8.

Edtorial . . . . . . . . . . 349

E. L. Hicks, H. JACKson, R. Elllis, on Herodas . . . . . . . . . 350

L. Campberl, on the Papyrus Fragment of the Phaedo . . . . . . . 363

A. C. Clark : The Library of J. G. Graevius . . . . . . . 365

W. M. Lindsay : Latin Accentuation . 373

J. Strachan : Ambulare . . . . . . 377

F. W. Thomas : Non, Noenum, N'́dvvos 377

Ellis's Noctes Manilianae. E. A. SonnENSCHEIN . . . . . . . . . . . . . 379

Hessels' Latin Anglo-Saxon Glossary. H. Nettleship . . . . . . . . . 382

Seyffert's Dictionary of Classical Antiquities. A. S. WILkins . . . . . 384

Weidner's Juvenal. E. G. Hardy . . 385

V. Henry's Comparative Grammar. P. GiLes

Paul's History of Language. $\dot{H}$. $\dot{D}$. Darbishike
Allen's Notes on Greek MSS. E. M. T. 387 Bayfield's Alcestis. W. S. HAdLeY . . 387 Notes.

Aesch. Agam. 557, 1118. 1125, 1227.

E. A. I. M. and H. L. B. . . . 388

Aesch. Supp. 819, 970 . C. J. Brennan 388

Soph. Trach. 504. L. H. S. . . . . 389

Plut. Them. 21. H. Richards . . . 389

Inscribed Whorl from Troy. A. $\mathrm{H}$.

SAYCE . . . . . . . 389

Herodas IV. A. S. Murnay . . . 389

Thuc. IV. 64. G. E. MARINDIN . 389

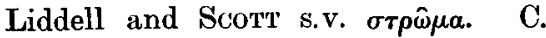

TorR . . . . . . . . . 390

Archaeology.

Monthly Record. H. B. Walteus . 390

Notes from Italy and Greece. H. A.

TubBs .......... . 393

Summaries . . . . . . . . . . 394

Periodicals . . . . . . . . . 395

Bibliography (English) . . . . . . 396 


\section{No. 9.}

E. B. Clapp, on Mr. Bayfield's Doctrine ${ }^{\text {Page }}$ of the Conditional Sentence . . . 397

E. W. B. Nicholson: Notes on Some Fragments of Menander . . . . 399

L. Campbell : Notes on the Antiope . 401

W. M. Lindsay : Latin Accentuation (concluded) . . . . . . . . . . 402

P. Schwenke: Apparatus Criticus to the Natura Deorum (continued) . . 408

Homeric Scholia (Schrader's Porphyrius on the Odyssey and Nicole's Geneva Scholia on the Iliad). W. LEAF . . 412

Plummer's Epistles of St. James and St. Jude. J. B. MaYor . . . . . . . 414

Wilcken's Greek Palaeography. E. M. Thompson . . . . . . . . . . . 417

Graux, Facsimiles of Greek MSS. in Spain. E. M. Thompson . . . . . 419

Liebenam on the History and Organization of the Roman Collegia. E. G. HARDY . . . . . . . . . 420

Freeman's History of Sicily Vols. I. and II. A. Houm . . . . . . . . 423 Smith's Dictionary of Antiquities. New Edition. J. E. Sandys . . . . . 425 Kempf's Valerius Maximus, H. T. РесK 428
PARE Hild's Juvenal VII. H. N. . . . . . 429 Foerster on the Physiognomia of Apuleius. H. N. . . . . . . . 429 Clarke's Evagoras of Isocrates. E. S. Thомpson . . . . . . . . . . . . 429 Sabrinae Corolla, ed. 4. E. D. S. . 430 Georges' Lexicon der Lateinischen Wortformen. H. N. . . . . . . 431 Graves' Burial Customs of the Ancient Greeks . . . . . . . . . . 431 Notes.

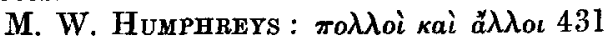

C. H. KeENE: Scholia on the Electra of Euripides . . . . . . . . .432

C. M. Mulvany : Notes on Propertius ............. 433

G. Mc. N. Rushforth: Tisidium . 433

J. E. SANDYS : Pronunciation of Suidas . . . . . . . . . . 434

F. W. Thomas : Suum Cuique . . 434

H. Richards : Herod. VIII. 111 . . 434 Archaeology.

Monthly Record . . . . . . . . .434

Theatre at Megapolis. C. T. . . . 437 Summaries of Periodicals . . . . . . 437 Bibliography (Foreign) . . . . .440
J. Adam : The Myth in Plato's Politicus 445

F. W. WALKER : Philological Notes . . 446

C. A. M. Fennelu: Brugmann's Theory of the Nasalis Sonans . . . . . . .451

L. Campbelx : On the Papyrus Fragment of the Phaedo.

R. Elus: On the Epoch of Herodas . 457

P. Schwenke: Apparatus Criticus to the Natura Deorum (concluded) . . . . 458

Wilamowitz-Moellendorff's Herakles. N. WEDD . . . . . . . . . . . 461 Recent Literature on the 'AOnvaíwv

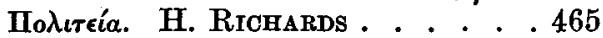
Cambridge Texts and Studies. Vol. I.

Part I. A. Plummer . . . . . . . . 468 Dyer's Studies of the Grods in Greece.

W. W. Fowler . . . . . . . . . 470

Keil's Edition of Varro's Res Rusticae.

H. Netrueship . . . . . . . . . . 474

Friedrich's edition of Cicero's Opera

Rhetorica. Vol. II. A. S. WiLkINs 475
G. M. Edwards's Iliad XXIII. A. Plate . . . . . . . . . . 476 Stein's Herodotus VII. R. W. MACAN 477 Xenophon's Memorabilia by Brietenbach and Mücke. P.G. ; by J. Marshall. E. S. Shuckburgh . . . . . . . 477 Holden's Cyropaedia. E. G. Coy . . 478 Stone's Anabasis IV. E. S. SnUckBURGH . . . . . . . . . . . 478 Dowdall's Hellenica I. A. H. Cooke . 478 Pearson's Fragments of Zeno and Cleanthes. H. N. FowLIR . . . . . . . 479 Notes.

Additional Fragments of Herodas. F. G. KenYon . . . . . . . . . 480 Corrected Readings of Papyrus. F. G. KenYoN . . . . . . . 482 The name Herodas in an Egyptian Inscription. F. G. KENYON . .. . 483 Notes on Herodas by W. R. Paton and A. C. Pearson . . . , . . 483 
Aristophanes Ach. $920-925$. C. J. ${ }^{\text {PAGE }}$ Brennan ........: 484 Ov. Trist. III. P. SANDFoRd . . . . 485 Juv. Sat. XI. 121. JoHN E. B. Mayon . . . . . . . . . 485 Scrupuli. H. D. Darbishire . . 485 Archaeology.

Monthly Record . . . . . . . 485
Plin. N. H. XXXV, 132. E. F Benson . . . . . . . . 486 Macmillan Lekythos. WATK Iss Luoyd 486 Ernst Curtius, Die Stadtgeschichte der Athen. E. Sellers . . . . . 486 Summaries of Periodicals . . . . . . . 487 Bibliography ........ . 490 